\title{
A new look at recognition in the Brown-Peterson distractor paradigm: Toward the application of new methodology to unsolved problems of recognition memory
}

\author{
VINCENT R. BROWN \\ Hofstra University, Hempstead, New York \\ and \\ DAVID S. GORFEIN \\ University of Texas, Arlington, Texas
}

\begin{abstract}
Data from a recognition version of the classic Brown-Peterson short-term memory paradigm was analyzed using a modified version of the conjoint recognition model (Brainerd, Reyna, \& Mojardin, 1999), which assumes that recognition is based on either a verbatim comparison of the recognition probe and the target item or a gist comparison of the items. Separate groups of participants were instructed to judge whether the recognition probe was an item from the current trial (exclusion condition), a previous trial (prior-only condition), or either the current or the previous trial (inclusion condition). The concept of gist is commonly thought of as meaning based. Our interpretation of the results suggests that the concept of gist need also emphasize similarity of environmental context. In addition, the results show that priming the recognition probe affects a participant's decision bias but does not enhance or impair the memory traces on which the recognition judgments are based. An additional analysis using Batchelder and Riefer's (1990) source-monitoring model supports the observation that priming affects only decision bias.
\end{abstract}

A considerable impetus to the current interest in recognition memory was supplied by Jacoby's (1991) attempt to develop a process-sensitive analysis of memory tasks. In this methodology, two or more lists are studied and followed by a recognition test under one of two post study instructions. Under what is labeled the exclusion instructions, participants are asked to say "old" to any item that was on one particular list (e.g., the first list studied) and "new" to any other word, including words presented on the other study list. Under inclusion instructions, participants are asked to say "old" to an item presented on any study list and "new" to any extra-experimental item. The methodology has the potential of separating recognition based on familiarity from recognition based on recollection (Mandler, 1980). Although the interpretation of the results from experiments in which Jacoby's process dis-

A previous version of this study was presented as a poster at the 2001 Annual Meeting of the Psychonomic Society in Orlando, FL. The authors thank Larry Carter and Kenya Malcolm, who helped with the collection of the data. We also thank Kay Erusu for her help in the preparation of the figures. The comments of Charles Brainerd, Robert Greene, William Hockley, and an anonymous reviewer contributed to the final version of this article. Correspondence may be addressed to either V. R. Brown, Department of Psychololgy, 135 Hofstra University, Hempstead, NY 11549 (e-mail: psyvrb@hofstra.edu) or D. S. Gorfein, Department of Psychology, University of Texas, Box 19528, Arlington, TX 760190528 (e-mail: gorfein@uta.edu). sociation technique has been used have been controversial (see Brainerd, Reyna, \& Mojardin, 1999, for a recent review), the methodology has been widely employed. Criticisms of the approach include the facts that the fit of the process dissociation model cannot be tested and the model contains no parameters for guessing bias.

In developing their fuzzy trace theory of memory, Reyna and Brainerd (1995) emphasized the distinction between verbatim recall and what is referred to as gist memory. They proposed that at encoding, two different kinds of memory traces are potentially formed. One, the verbatim trace, is seen as an exact representation of the memory event. The other, the gist trace, records only certain general features of the event, such as its meaning. According to Reyna and Brainerd, the accessibility of the verbatim trace declines rapidly, whereas the availability of the gist trace declines very slowly. In an effort to separate verbatim memory from gist memories, Brainerd et al. (1999) have developed a variation of Jacoby's (1991) exclusioninclusion procedure. In this paradigm, a single list of words is presented for study. The recognition test list, however, contains three types of items: the study items, items semantically related to the study items, and novel distractors. Prior to recognition testing, participants are presented with one of three sets of instructions: (1) accept any item that was on the study list, (2) accept only items that appear to be related to the studied items (items rec- 
ognized as old are to be rejected), or (3) accept any item that was on the study list and any item that appears to be related to a list item. Instructions 1 and 3 are similar to Jacoby's exclusion and inclusion instructions, respectively, whereas Instruction 2 (called related instructions by Brainerd et al., 1999) is a new condition designed to explicitly tap familiarity- or gist-based processing. The accuracy data generated from this $3 \times 3$ design have nine degrees of freedom and, therefore, can be used to test models with up to eight free parameters. It is the fact that Brainerd et al.'s (1999) model has fewer parameters than the degrees of freedom available in the data that gives it an advantage over Jacoby's original process dissociation model, in which the number of model parameters exhausted the available degrees of freedom, making it impossible to test the fit of the model. The new model also explicitly includes response bias (guessing) parameters, an important addition to Jacoby's model.

Brainerd et al.'s (1999) model, which they called the conjoint recognition model, is an example of a type of model that has recently become popular in cognitive psychology. Multinomial models provide an important alternative or supplement to standard "off-the-shelf" approaches to data analysis, such as the analysis of variance (ANOVA). When available, model-based approaches are preferred, because they explicitly incorporate the psychological processes assumed to be operating in the task that gave rise to the data. The interpretation of standard statistical analyses can be problematic, because it is not always clear how the underlying psychological processes give rise to the overt measured responses (Batchelder \& Riefer, 1990; Brainerd et al., 1999; Riefer \& Batchelder, 1988). Among the variety of model-building approaches, multinomial models have the advantage of being relatively easy to formulate and analyze. The techniques for designing and testing such models are well understood (Riefer \& Batchelder, 1988). Multinomial models simplify the underlying psychology by treating processing as a discrete set of operations proceeding in distinct stages. What happens in each stage is contingent on the outcome of previous stages. Although the assumption of discrete sequential processing stages may seem restrictive, multinomial models are actually quite flexible. In fact, parallelprocessing assumptions can be incorporated into the hierarchical model structure (e.g., V. Brown, 1998). Since the landmark article by Riefer and Batchelder, multinomial models have been developed to study a number of different paradigms in cognition and perception (see Batchelder \& Riefer, 1999, for a review).

In examining these new methodologies, we recognized that the participant in the classic Brown-Peterson task is essentially given exclusion instructions (i.e., distinguish the current to-be-remembered item, or TBRI, from previously presented items). The Brown-Peterson shortterm memory (STM) task requires an individual to remember a number of items for a matter of seconds, while occupied by a rehearsal-preventing task (J. A. Brown, 1958; Peterson \& Peterson, 1959). It is the fact that a typical
Brown-Peterson experiment consists of long sequences of trials run in immediate succession that forces the participants to have to distinguish the items presented on the current trial from those presented on previous trials.

Although the vast majority of studies employing the Brown-Peterson task have required recall of the TBRIs, a very few studies have employed a recognition procedure (Fisher, Jarombek, \& Karsh, 1974). Among these, Gorfein and Jacobson (1972) used a multiple-choice procedure to demonstrate the existence of proactive interference in the recognition task. Bennett (1975) employed a two-alternative forced-choice procedure, in which the false choice had been a TBRI on an earlier trial, to demonstrate that the probability of correctly selecting the current TBRI was greater when the foil was of greater lag. Gorfein and Jacobson (1973) employed a yes-no decision where the TBRI was a single word and the recognition probe was either that word or a false lure that was either a word that had been a TBRI on a prior trial or a word new to the experiment. Both decision latency and errors increased over trials when the words were from the same taxonomic category but returned to the trial one level when the semantic category was changed, indicating the build-up and release of proactive interference.

Gorfein (1987) reviewed the literature and provided a theoretical synthesis of research in the Brown-Peterson paradigm. He suggested that recall and recognition of a TBRI are strongly influenced by two kinds of context: environmental and semantic. With respect to environmental context, following the general outline of Estes's (1950) stimulus-sampling theory, it was suggested that the context of each TBRI was encoded with the current stimulus sample and that context fluctuated over time. Retrieval and recognition were based on the similarity of the context at test to that at encoding. Crowder and Greene (1987) have pointed out the similarity between Gorfein's notion of environmental context and Glenberg's (e.g., 1987) notion of temporal context, as put forth in his temporal distinctiveness explanation of long-term recency. Semantic context referred to the relationship in meaning among the TBRIs as demonstrated in studies showing a build-up of proactive interference with repeated taxonomic categories and a release from interference when the category is changed (Gorfein \& Jacobson, 1973; Loess, 1967; Wickens, Born, \& Allen, 1963). Gorfein proposed two roles for semantic context, one in enhancing the activation of the TBRIs and the other in influencing the similarity of same-category TBRIs. In the present study, we chose to minimize the additional complications produced by semantic similarity and employed unrelated words as TBRIs. This is a distinct variation from the original application of the concept of gist processing. The emphasis in the present study is thus on the role of the environmental context, rather than on that of the semantic context.

Once we saw the similarity of the exclusion instructions for the conjoint recognition model to those employed in the recognition version of the Brown-Peterson task, it seemed that the conjoint recognition model could 
be borrowed to analyze the processes underlying performance in the Brown-Peterson recognition task. Specifically, we employ the yes-no recognition procedure of Gorfein and Jacobson (1973) under three different instructional conditions: (1) say "yes" to the target only if it is the current TBRI (exclusion), (2) say "yes" to any target that has been presented as a TBRI, but not to the current trial TBRI (prior only), and (3) say "yes" to any target that has been presented as a TBRI in the experiment (inclusion). We believe that the prior-only instruction and the use of prior items as foils in lieu of the highly related items employed by Brainerd et al. (1999) places a different emphasis on the concept of gist and implies that the term gist should be broadened to explicitly include elements of the environmental/temporal context of a stimulus, in addition to the semantic context. In the prior-only condition, participants are required to discriminate prior from current TBRIs, and the defining difference between the two is the trial on which the item first appeared: Since none of the presented items are semantically related, similarities or differences in meaning cannot be the primary basis for this discrimination.

To examine whether performance in the recognition task is consistent with the contextual fluctuation explanation, we included two levels of prior probes as foils for recognition-prior TBRIs drawn from the immediately preceding trial and prior TBRIs drawn from the item presented two trials back - and we primed the current target item on half of the trials. According to the contextual fluctuation interpretation offered by both Bennett (1975) and Gorfein (1987), the parameters of the modified conjoint recognition model associated with distinguishing prior from current TBRIs should reflect the results of the lag manipulation. Again, it should be noted that in this task, gist is manipulated and interpreted differently from that in Brainerd et al. (1999), in that semantic similarity is minimized by the use of unrelated items and it is only their occurrence in the experimental context that makes the prior items more similar to the current TBRI than the new items used as alternative probes.

On primed trials, the recognition probe was preexposed in lowercase letters for $50 \mathrm{msec}$ between the fifth and sixth number to be shadowed during the retention interval. The brief exposure of the prime was intended to be of sufficient duration to be seen but fast enough so that the connection between the lowercase prime and the uppercase target was not obvious. We employed the prime to see whether the prime would influence the speed and accuracy of the recognition decision. An important advantage of the conjoint recognition model is that it allows us to distinguish the locus of priming effects. Much research employing priming in recognition memory has suggested that the principal effect of briefly presented primes is to influence response bias (Bernstein \& Welch, 1991; Jacoby \& Whitehouse, 1989). Alternatively, the prime could result in the attachment of the context at the time of the prime to the internal representation of the target.
The recognition version of the STM task requires that the participant differentiate among similar recent events. Under exclusion (current TBRI only) instructions, the current TBRI must be distinguished from foils drawn from immediately preceding items that were presented in a highly similar context, and under prior-only instructions, recently presented TBRIs must be distinguished from the just-presented current TBRI. To the extent that priming produces a feeling of familiarity (Ratcliff \& McKoon, 1997) and/or increases perceptual fluency (Bowers, 1999), we expected that priming would have no influence on decision accuracy under exclusion and prioronly instructions, which require differentiation among recent and, therefore, presumably familiar probes. Only the inclusion instruction should show the influence of the priming manipulation, since this condition requires discrimination between old and new items, but not discrimination among the old items (current vs. prior). Thus, although perceptual fluency may increase performance in certain implicit memory experiments (e.g., Zeelenberg, Wagenmakers, \& Raaijmakers, 2002), the need to differentiate among relatively similar traces precludes its making a contribution to the accuracy performance under the exclusion and prior-only instructions in the present experiment; participants should quickly recognize that familiarity is an insufficient basis for a response, since one third of the foils have also occurred in the experiment and they are trying to avoid saying "yes" to them.

The priming manipulation in the STM task is, therefore, unlike conditions in more typical tests of priming in memory. Such tasks as list learning followed by recognition testing for old and new items and continuous recognition tend to encourage the use of familiarity as a means for responding, and eliminating the familiarity bias requires directly instructing participants that a prime is being presented or the presentation of a prime that is easily seen (e.g., Jacoby \& Whitehouse, 1989). With the present procedure, we could examine the effects of priming without the need to specifically call attention to the primes.

Only in the event that the prime directly influences information with respect to recency of the item's memory trace would we expect an influence on accuracy. Therefore, the inclusion of the priming manipulation in the present study can be viewed as an attempt to differentiate models of priming in memory tasks that attribute prime influence to a bias induced by a feeling of familiarity from models that postulate that a prime is encoded in a manner similar to a TBRI.

A graphic representation ${ }^{1}$ of the conjoint recognition model, as modified for the present task, is shown in Figure 1. This model assumes that participants' responses are based on either a verbatim judgment (V) or a gist judgment (G). Experiment participants are assumed to base their decision about the recognition items on the verbatim trace with a probability of $V$. Stronger verbatim traces would be reflected by higher values of $V$. Verbatim judgments are expected to vary as a function of lag, so three parameters are needed: $V_{\mathrm{C}}, V_{\mathrm{L} 1}$, and $V_{\mathrm{L} 2}$ (verbatim 


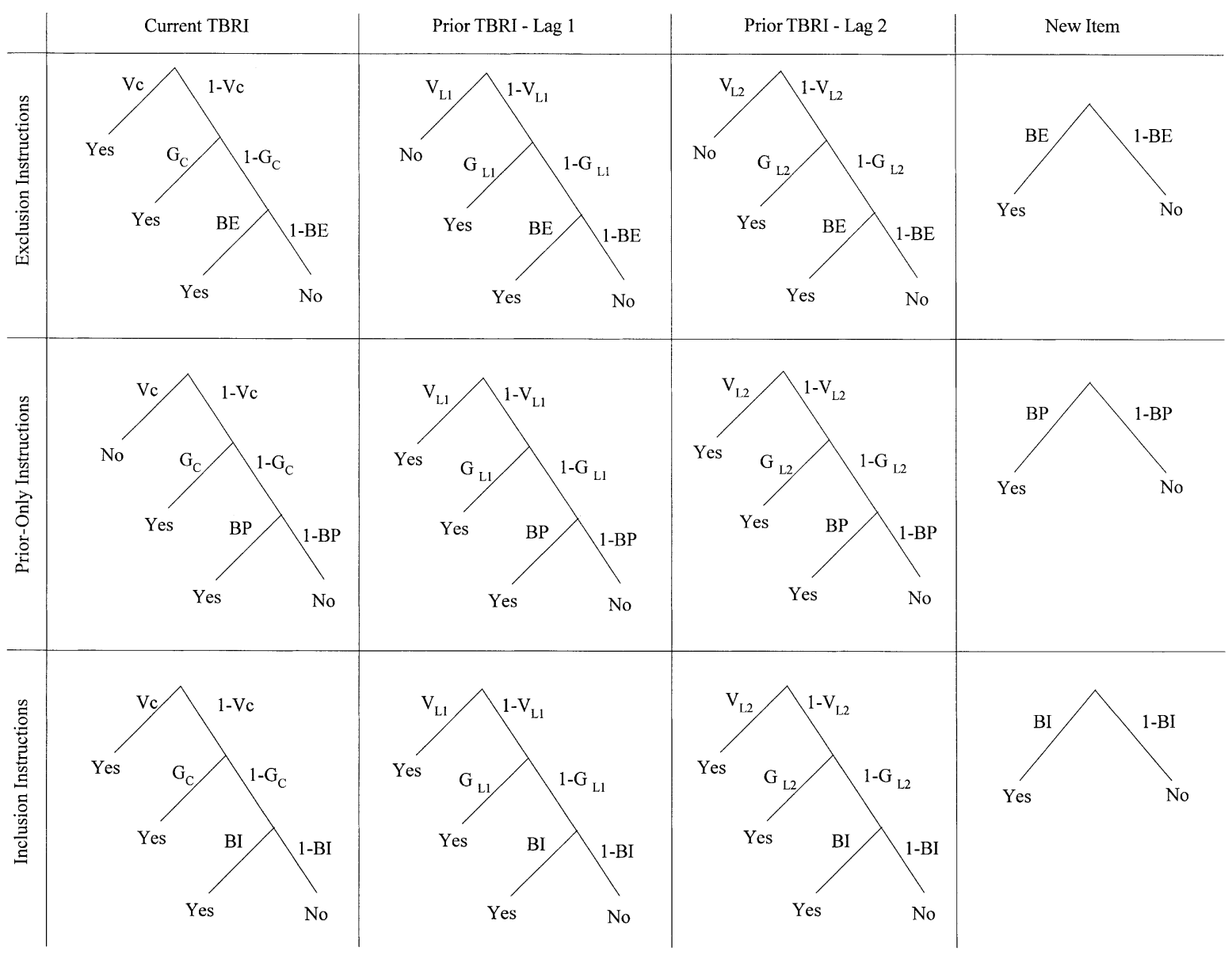

Figure 1. Tree diagram for the conjoint recognition model. $V_{\mathrm{C}}$, probability of verbatim recognition of the current to-be-remembered item (TBRI); $V_{\mathrm{L} 1}$ and $V_{\mathrm{L} 2}$, probability of verbatim recognition of the prior TBRIs at lag 1 and lag 2 ; $G_{\mathrm{C}}$, probability of gist recognition of the current TBRI; $G_{\mathrm{L} 1}$ and $G_{\mathrm{L} 2}$, probability of gist recognition of the lag1 and lag 2 TBRIs; $B E$, probability of guessing "yes" under exclusion instructions; $B P$, probability of guessing "yes" under prior-only instructions; $B I$, probability of guessing "yes" under inclusion instructions.

current, verbatim lag 1 , and verbatim lag 2 , respectively). ${ }^{2}$ If the verbatim trace is not retrieved or is insufficient for making a decision (with a probability of $1-V$ ), the decision is based on the gist trace with a probability of $G$. The gist trace is also predicted to differ as a function of lag, so the gist parameters are $G_{\mathrm{C}}$ (gist current), $G_{\mathrm{L} 1}$, and $G_{\mathrm{L} 2}$ (gist lag 1 and gist lag 2, respectively).

In the case of our revised procedure, we would expect context to affect the two memory parameters of the model, $V$ and $G$. To the extent that the contextual fluctuation view holds, we would anticipate that the context at the time of retrieval would show decreasing resemblance to the encoded context for any TBRI as a function of the time elapsed since the initial presentation of that TBRI. Both the $V$ and the $G$ parameters should decrease as a function of that time interval. Therefore, it is expected that $V_{\mathrm{C}}>$ $V_{\mathrm{L} 1}>V_{\mathrm{L} 2}$ and $G_{\mathrm{C}}>G_{\mathrm{L} 1}>G_{\mathrm{L} 2}$. Also, since the verbatim trace is assumed to decline more rapidly than the gist trace, the difference between $V_{\mathrm{C}}$ and $V_{\mathrm{L} 1}$ should be greater than the difference between $G_{\mathrm{C}}$ and $G_{\mathrm{L} 1}$. If the prime results in the attachment of the context at the time of the prime to the internal representation of the target, the effect will be to increase the $V$ and $G$ parameters of the conjoint recognition model; otherwise, the prime should not affect the memory parameters, but only, perhaps, the bias parameters.

Finally, when presented with novel distractors, the participant is assumed to occasionally confuse these distractors with either a current or a prior item. The probability of this happening is the model's measure of response bias, which is assumed to depend on the task instructions, because the three instructions require participants to make different discriminations (i.e., exclusion and prior-only instructions require a discrimination among previously presented items, whereas inclusion instructions require the participant only to distinguish presented items from never-presented items). So there are three guessing parameters: BE (bias exclusion), BP (bias prior only), and $\mathrm{BI}$ (bias inclusion). In the event that both the verbatim and the gist traces are insufficient to make a decision [which occurs with a probability of $(1-V) *(1-G)]$, the par- 
ticipant is assumed to guess with a probability of $\mathrm{BE}, \mathrm{BP}$, or BI, depending on their task instructions. In typical list memory applications, the instructions are given following list learning, rendering learning potentially equivalent across instructional conditions. In the STM procedure, the participant must be preinstructed as to the decision task. This creates the possibility that different instructions can produce different learning strategies. The conjoint recognition model has the potential to separate learning differences from bias effects of the instructions.

We also noted a high degree of similarity between the contextual fluctuation perspective and what has become known as source monitoring (Johnson \& Raye, 1981). The present implementation of the Brown-Peterson recognition paradigm requires participants to implicitly identify the source of the recognition probes. We therefore decided to evaluate this interpretation by fitting Batchelder and Riefer's (1990) multinomial source mon- itoring model to our data. Our modification of Brainerd et al.'s (1999) procedure requires the participant to correctly discriminate the target source in two of the three instruction conditions (exclusion and prior only). The exclusion condition requires the target item either to be classified as the current TBRI or not. The prior-only condition can be thought of as requiring the labeling of the target item as originating from one of three sources: the most recent trial, an earlier trial, or neither. Our approach differs from the standard source-monitoring approach in that in source-monitoring experiments, the participant is typically given explicit instructions to distinguish between specific contexts, such as indicating whether an item appeared first in a visual or an auditory context.

A graphic representation of the source monitoring model is shown in Figure 2. The model tested here is a modified version of Batchelder and Riefer's (1990) twosource model. The original model has parameters for item detection $(D)$, source identification $(S)$, and guess-

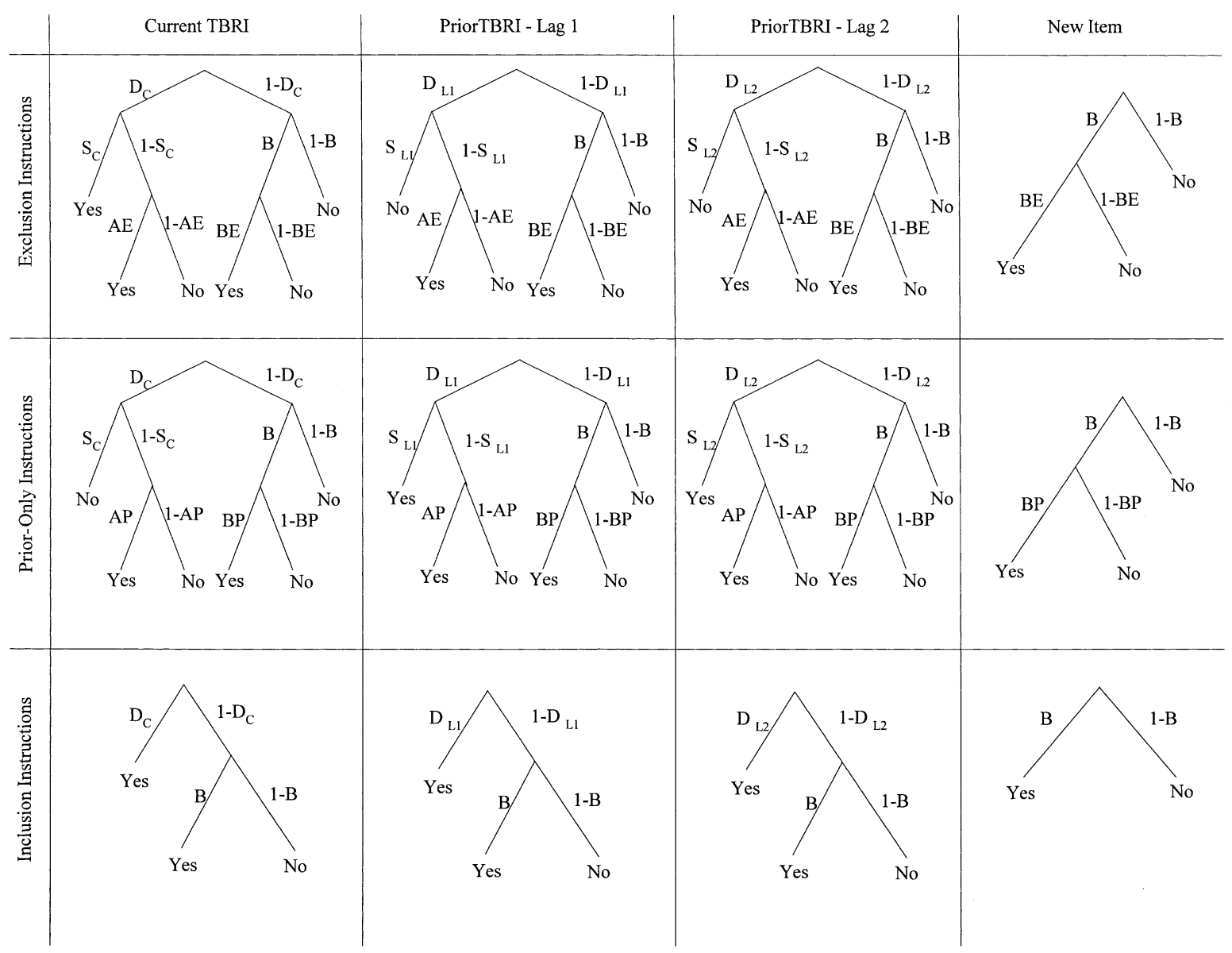

Figure 2. Tree diagram for the source-monitoring model. $D_{\mathrm{C}}$, probability of distinguishing the current TBRI from a new item; $D_{\mathrm{L} 1}$ and $D_{\mathrm{L} 2}$, probability of distinguishing lag 1 and lag 2 TBRIs from new items; $S_{\mathrm{C}}$, probability correctly identifying the current TBRI as current; $S_{\mathrm{L} 1}$ and $S_{\mathrm{L} 2}$, probability correctly identifying lag 1 or lag 2 items as prior; $B$, probability of guessing that an item is not new (i.e., is either a current or prior TBRI); $B E$, probability of guessing "yes" under exclusion instructions when an old/new discrimination has not been made; $B P$, probability of guessing "yes" under prior-only instructions when an old/new discrimination has not been made; $A E$, probability of guessing "yes" under exclusion instructions when the source (current or prior) has not been identified; $\boldsymbol{A P}$, probability of guessing "yes" under prior-only instructions when the source (current or prior) has not been identified. 
ing biases. In a standard two-source experiment, participants are given a recognition test in which they are asked to indicate which of two sources the presented item came from or whether it is a new item. This procedure utilizes three types of test items and a single set of instructions. On the other hand, the modified Brown-Peterson paradigm used here presents participants with four types of probe items (current, prior lag 1, prior lag 2, and new) under three sets of instructions (exclusion, prior only, and inclusion). In the most general case, a separate version of the model would be required for each set of instructions. However, since the full two-source model has seven parameters, requiring a unique set of parameters for each of the three sets of instructions would create a 21-parameter model, more than the 12 degrees of freedom ( 3 instruction sets $\times 4$ probe types: current, lag 1, lag 2 , and new) available to test the model. We reduce the size of the model by assuming that participants' ability to detect whether an item is old or new and to identify the source of the item (current or prior) is independent of the instructions they are given. Clearly, participants' tendency to guess (response bias) will be a function of the specific responses they are required to make; thus, the response bias parameters will, in general, be a function of the instruction condition, as they are in the conjoint recognition model. The parameters of the modified source-monitoring model are listed in the caption to Figure 2.

With respect to the contextual fluctuation theory, we expect that the primary effect of the manipulation of target lag would be on the two source memory parameters $D$ (the probability of discriminating a presented item from a new item) and $S$ (the probability of assigning a discriminated item to the appropriate category, current or prior). Both parameters are expected to decrease as a function of lag. If priming affects the memory trace itself, the $D$ and $S$ parameters should be affected by the priming manipulation, but if priming serves only to alter a participant's response strategy, the guessing parameters will be affected, but the $D$ and $S$ memory parameters should be unaffected by the priming manipulation.

\section{METHOD}

\section{Participants}

Thirty-six students at the University of Texas-Arlington served in this study to satisfy a course requirement. The participants were assigned to one of three instructional conditions in a counterbalanced order.

\section{Design}

The recognition latency version (Gorfein \& Jacobson, 1973) of the Brown-Peterson distractor task was employed. In this procedure, a single word was presented as a TBRI on each trial. Following a 6sec interval filled with a number-reading task, one of four possible single-word target types was presented for a recognition decision. The target was either the current trial TBRI or the TBRI from the immediately prior trial (lag 1), the TBRI from two trials back (lag 2), or a word not previously presented in the experiment. Half of the target trials were primed by presenting the target word briefly during the retention interval. Three different instructional conditions were varied between subjects: exclusion, prior only, and inclusion. Thus, the study employed a mixed-model factorial design: 3 instructions $\times 4$ target types $\times 2$ levels of priming (primed vs. unprimed targets). The experimental design is depicted in Table 1.

\section{Materials}

Two word lists were constructed. The materials were chosen at random from among 355 high-frequency four-letter English nouns, with the restriction that there were no obvious associations between adjacent TBRIs. The materials were used in the order chosen, with the first 10 words serving as a practice list. The next 6 words served as a warm-up buffer, with Items 5 and 6 also serving as targets of lags 1 and 2 for the first 2 test words. The next 144 words (Trials 7-150) were selected as TBRIs. The choice of target type for recognition was randomly assigned, with 48 trials probed with the current TBRI (current), 24 probes were drawn from one trial back (lag 1) and 24 from two trials back (lag 2), and 48 additional words were drawn from the remaining items of the original list (new). Half of each target type was randomly assigned to the primed condition, and these items were counterbalanced across participants. The counterbalancing of primes resulted in two unique lists, which were used equally often in each instructional condition.

\section{Procedure}

The sequence of trial events is presented in Table 2. The participants were individually tested with items presented centered on a computer monitor and timed using the MEL2 system (Schneider, 1988). A single trial consisted of a 500-msec ready signal (\&\&\&\&), a 250 -msec presentation of the TBRI in uppercase letters, a 6-sec filled retention interval, a target probe presented in uppercase letters until response or a maximum of 3,000 $\mathrm{msec}$ (if the response time [RT] was less than $3,000 \mathrm{msec}$ the screen was blanked for the remainder of the 3,000-msec interval), accuracy feedback for $200 \mathrm{msec}$, and finally, a 1,800-msec timeout (****). Each trial was, therefore, $11,750 \mathrm{msec}$ in duration.

The retention interval activity was number shadowing, with the participant asked to read aloud a series of six four-digit numbers as single digits - for example, 7456 was to be read as "seven, four, five, six." The numbers were sampled on each trial from the digits $0-9$, without replacement. Each four-digit number was presented for $950 \mathrm{msec}$ and was followed by a 50 -msec blank field. On primed trials, the prime was presented in lowercase letters during the $50 \mathrm{msec}$ following the fifth four-digit number. The prime, therefore, was partially masked by the prior and the subsequent digit sets.

Table 1

Experimental Design/Response Instructions

\begin{tabular}{lcccc}
\hline & \multicolumn{4}{c}{ Target } \\
\cline { 2 - 4 } Instructions (Between Subjects) & Current TBRI & Lag1 TBRI & Lag 2 TBRI & New Item \\
\hline Current item (exclusion) & say "yes" & say "no" & say "no" & say "no" \\
Prior item & say "no" & say "yes" & say "yes" & say "no" \\
Either current or prior (inclusion) & say "yes" & say "yes" & say "yes" & say "no" \\
\hline
\end{tabular}

Note-TBRI stands for to-be-remembered item. 
Table 2

A Gorfein-Jacobson Recognition Trial (Unprimed)

\begin{tabular}{llc}
\hline \multicolumn{1}{c}{ Trial Event } & Example & Duration $(\mathrm{msec})$ \\
\hline Ready signal & $\& \& \& \&$ & 500 \\
To-be-remembered item & moon & 250 \\
First number to be shadowed & 7456 & 950 \\
Blank & & 50 \\
$\ldots$ & $\ldots$ & $\ldots$ \\
Sixth number to be shadowed & 2965 & 950 \\
Blank & & 50 \\
Target item & barn & 3,000 \\
Feedback & error & 200 \\
Intertrial interval & $* * * *$ & 1,800 \\
\hline
\end{tabular}

Note-On primed trials, the target item is presented in lowercase for $50 \mathrm{msec}$ between the fifth and the sixth number sets (i.e., instead of the 50-msec blank interval).

Instructions (see below) were given by the experimenter, and these were followed by a 10 -trial practice block. The practice block included all four target types. No primes were presented during the practice block, and no mention was made of the possibility of the primes appearing. During practice, the experimenter clarified the error feedback in accord with the instructional condition. The participant was then left to complete the 150 test trials. The numbershadowing task was tape recorded to ensure that the participant fully engaged in the rehearsal-preventing task. At the end of the session, the participants completed a brief postexperimental questionnaire designed to assess whether the participant saw the prime and whether its connection with the target was noticed.

\section{Instructions}

The three tasks had some common instructions and some unique to the task. All the participants were instructed to respond as quickly as they could, consistent with being correct. They were to keep their fingers resting on the number pad of the computer keyboard, with one finger resting on the yes (1) key and another on the no (2) key. All the individuals were shown a demonstration trial and were told that following the ampersands, which served as a warning signal, they would see a single word, which they were to read to themselves and try to remember. The to-be-remembered word would always precede the onset of a series of four-digit numbers, which they were to read aloud. The participants' reading of the numbers was tape recorded. The participants were not informed about the kinds of probes they would see, and no mention was made of the priming events.

The specific instructions for the recognition probe were then given, and the nature of the feedback and brief rest were also explained.

Exclusion condition (standard Gorfein and Jacobson instructions). The participants were instructed to press the "yes" key only if the probe item was the current trial item and to press the "no" key for any other word.

Prior-only condition. The participants were told to press the "yes" key if the probe was any item they had been asked to remember in the experiment other than the current trial item. They were instructed to press the "no" key for the current item and any item that had not been presented as a to-be-remembered word.

Inclusion condition. The participants were instructed to press the "yes" key if the probe item was either the current TBRI or an item that they had been asked to remember on any previous trial. They were instructed to press the "no" key if the probe had not been an item that they had previously been asked to remember.

\section{RESULTS}

The postexperimental questionnaire revealed that all the participants in the primed condition could report that they had seen a word on one or more trials among the numbers they were shadowing. Their estimate of the number of these events (actually, 72) was predominately between 10 and 30 . No one described the contingency between the prime and the target, although a few may have recognized it.

The mean proportions of correct responses and mean RTs are presented in Table 3. Separate ANOVAs were performed for the accuracy and RT data. For both analyses, alpha was set at .05.

\section{Accuracy}

The ANOVA of the accuracy data indicated an interaction between the three experimental variables of instruction, priming, and type of target $[F(6,99)=2.32$, $\left.M S_{\mathrm{e}}=0.0156\right]$. In addition to the significant three-way interaction, two of the three two-way interactions reached significance [instructions $\times \operatorname{target} F(6,99)=4.55, M S_{\mathrm{e}}=$ 0.0316 ; prime $\times$ target, $F(3,99)=0.0116]$. The instructions $\times$ prime interaction $\left[F(2,33)=3.02, M S_{\mathrm{e}}=0.0106\right.$, $p<.07]$ fell only marginally short of significance.

This complex set of interactions would seem to indicate that the effect of the prime depends on which specific combination of instruction and probe type is involved. Rather than describe these effects in detail, we reserve discussion pending our fitting of these data to the two multinomial models. However, we note that the proportion of correct responses show a clear effect of the prime in the inclusion condition. Priming appears to greatly increase the false alarms (i.e., there are fewer correct rejections) to new items in the inclusion condition. We also note the fact that in the prior-only condition, the false alarm rate to current probes is noticeably lower than the false alarm rate to new items. This result is inconsistent with a single-process explanation of the memory decision (e.g., one based solely on the similarity between distractors and targets) and can be explained only by a two-trace theory, such as the verbatim/gist distinction of fuzzy trace theory underlying the present analysis (Brainerd, Reyna, \& Kneer, 1995). ${ }^{3}$

The only significant main effect was that of instructions $\left[F(2,33)=5.15, M S_{\mathrm{e}}=0.0889\right]$; overall, correct responses were highest under the exclusion (current TBRI only) instructions. Notably, the main effect of priming 
Table 3

\begin{tabular}{|c|c|c|c|c|c|c|}
\hline \multicolumn{7}{|c|}{$\begin{array}{c}\text { Proportions of Correct Responses and Mean Reaction Times (RTs, in } \\
\text { Milliseconds) for Correct Recognition Decisions as a Function of } \\
\text { Instructions and Type of Recognition Target }\end{array}$} \\
\hline \multirow{2}{*}{$\begin{array}{l}\text { Target } \\
\text { Expected Response) }\end{array}$} & \multicolumn{2}{|c|}{ Unprimed } & \multicolumn{2}{|c|}{ Primed } & \multicolumn{2}{|c|}{ Mean } \\
\hline & $P$ & RT & $P$ & RT & $P$ & RT \\
\hline \multicolumn{7}{|c|}{ Exclusion Instructions } \\
\hline rrent (yes) & .80 & 1,013 & .82 & 999 & .81 & 1.006 \\
\hline g 1 (no) & .84 & 1,079 & .83 & 1,080 & .84 & 1,080 \\
\hline og 2 (no) & .88 & 1,080 & .87 & 1,025 & .88 & 1,052 \\
\hline lew (no) & .96 & 967 & .94 & 949 & .95 & 958 \\
\hline$M$ & .87 & 1,035 & .86 & 1,013 & .87 & 1,024 \\
\hline \multicolumn{7}{|c|}{ Prior-Only Instructions } \\
\hline urrent (no) & .82 & 1,028 & .84 & 871 & .83 & 949 \\
\hline og 1 (yes) & .67 & 1,156 & .76 & 990 & .72 & 1,073 \\
\hline og 2 (yes) & .69 & 1,170 & .69 & 964 & .69 & 1,067 \\
\hline lew (no) & .70 & 1,159 & .71 & 1,064 & .70 & 1,116 \\
\hline$M$ & .72 & 1,128 & .75 & 972 & .74 & 1,050 \\
\hline \multicolumn{7}{|c|}{ Inclusion Instructions } \\
\hline urrent (yes) & .85 & 1,026 & .86 & 939 & .86 & 983 \\
\hline og 1 (yes) & .76 & 1,062 & .76 & 1,051 & .76 & 1,056 \\
\hline og 2 (yes) & .74 & 1,100 & .78 & 1,050 & .76 & 1,075 \\
\hline lew (no) & .76 & 1,158 & .55 & 1,162 & .66 & 1,160 \\
\hline$M$ & .78 & 1,086 & .74 & 1,050 & .76 & 1,068 \\
\hline
\end{tabular}

was not significant, supporting the observation that under the conditions of this experiment, priming does not seem to influence the memory trace itself.

\section{Response Time}

The analysis of RT was conducted even though the decision required for each target type varied with instructions. The mean RTs are reported in Table 3. The antilog of the mean log RT for correct responses for each participant in each condition served as the basis for analysis. The ANOVA indicated an effect of priming $[F(1,33)=$ 9.16, $\left.M S_{\mathrm{e}}=39,740\right]$, with the mean recognition RT to primed targets $(1,012 \mathrm{msec})$ being lower than the mean RT to unprimed targets $(1,083 \mathrm{msec})$. The type of target also influenced the RT $\left[F(3,99)=9.40, M S_{\mathrm{e}}=15938\right]$, with the current TBRIs (979 msec) being recognized more quickly than the prior TBRIs $(1,067 \mathrm{msec})$ and the new items $(1,078 \mathrm{msec})$. There was no main effect of instructional task $\left[F(2,33)<1, M S_{\mathrm{e}}=340,183\right.$; mean exclusion $\mathrm{RT}=1,024 \mathrm{msec}$, mean prior- only $\mathrm{RT}=1,050$, mean inclusion RT $=1,068]$. The lack of a main effect of the instructions manipulation provides some support for the simplifying assumption made earlier that instructions condition does not affect memory retrieval processes.

The instructions variable, however, was involved in two significant two-way interactions. Conceptually, the clearer of these was the priming $\times$ instruction interaction $[F(2,33)=3.52]$. The effect of the prime was to decrease RT by about $22 \mathrm{msec}$ in the exclusion condition, $156 \mathrm{msec}$ in the prior-only condition, and $36 \mathrm{msec}$ in the inclusion condition. Table 3 shows that priming speeds things up across the board in the prior-only condition, speeds things up only for current items in the in- clusion condition, and has relatively little effect in the exclusion condition.

The interaction of the instructions variable and the type of recognition target $[F(6,99)=5.13]$ is only a little more difficult to describe. In all the instructions conditions, the lag 1 and lag 2 RTs differed very little (mean absolute difference is $17 \mathrm{msec}$ ) but were much longer, on average, than the responses to the current target $(88 \mathrm{msec})$. Although the response required to new probes was always "no," the pattern of RT to this target type changed markedly as a function of instructions. In the exclusion condition, the RT to new items was 48 msec shorter than even the RT to the current probes, whereas the RT to new items was $43 \mathrm{msec}$ longer than the longer of the two lags in the prior-only condition and $85 \mathrm{msec}$ longer than the longer of the two lags the longer of the two lags in the inclusion condition. Responses to current items are fastest in the prior-only and inclusion conditions, and responses to new items are fastest in the exclusion condition. No other effect approached significance.

The latency data suggest that increased latencies are associated with the locus of a signal detection threshold as a function of instructions. Specifically, we might postulate four distributions of item strengths, corresponding to the four probe types employed in this experiment: current, lag 1, lag 2, and new, in order of decreasing mean strength. Under any instructions condition, we would anticipate that when the probe presented was close to the placement of the threshold, responses would be slower than when it was a distance away from the critical cutoff. Therefore, for exclusion (current-only) instructions, the cutoff would be set to maximize correct responding and, thus, would occur between the mean 
Table 4

Parameter Estimates for the Full Conjoint Recognition Model

\begin{tabular}{lccccccccc}
\hline & $V_{\mathrm{C}}$ & $V_{\mathrm{L} 1}$ & $V_{\mathrm{L} 2}$ & $G_{\mathrm{C}}$ & $G_{\mathrm{L} 1}$ & $G_{\mathrm{L} 2}$ & $B E$ & $B P$ & $B I$ \\
\hline Unprimed & .67 & .47 & .51 & .37 & .27 & .21 & .04 & .28 & .25 \\
Primed & .69 & .48 & .44 & .30 & .29 & .20 & .06 & .29 & .44 \\
\hline
\end{tabular}

Note- $V_{\mathrm{C}}$, probability of verbatim recognition of the current TBRI; $V_{\mathrm{L} 1}$ and $V_{\mathrm{L} 2}$, probability of verbatim recognition of prior TBRIs at lag 1 and lag $2 ; G_{\mathrm{C}}$, probability of gist recognition of the current TBRI; $G_{\mathrm{L} 1}$ and $G_{\mathrm{L} 2}$, probability of gist recognition of the lag1 and lag 2 TBRIs; $B E$, probability of guessing "yes" under exclusion instructions; $B P$, probability of guessing "yes" under prior-only instructions; $B I$, probability of guessing "yes" under inclusion instructions.

Table 5

Parameter Estimates for the Full Source Monitoring Model

\begin{tabular}{lccccccccccc}
\hline & $D_{\mathrm{C}}$ & $D_{\mathrm{L} 1}$ & $D_{\mathrm{L} 2}$ & $S_{\mathrm{C}}$ & $S_{\mathrm{L} 1}$ & $S_{\mathrm{L} 2}$ & $A E$ & $A P$ & $B E$ & $B P$ & $B$ \\
\hline Unprimed & .80 & .67 & .65 & .88 & .76 & .82 & 1.0 & 1.0 & .04 & .28 & .25 \\
Primed & .79 & .63 & .58 & .60 & .78 & .84 & .97 & .39 & .06 & .29 & .44
\end{tabular}

Note $-D_{\mathrm{C}}$, probability of distinguishing the current TBRI from a new item; $D_{\mathrm{L} 1}$ and $D_{\mathrm{L} 2}$, probability of distinguishing lag 1 and lag 2 TBRIs from new items; $S_{\mathrm{C}}$, probability correctly identifying the current TBRI as current; $S_{\mathrm{L} 1}$ and $S_{\mathrm{L} 2}$, probability correctly identifying lag 1 or lag 2 items as prior; $A E$, probability of guessing "yes" under exclusion instructions when the source (current or prior) has not been identified; $A P$, probability of guessing "yes" under prior-only instructions when the source (current or prior) has not been identified; $B E$, probability of guessing "yes" under exclusion instructions when an old/new discrimination has not been made; $B P$, probability of guessing "yes" under prior-only instructions when an old/new discrimination has not been made; $B$, probability of guessing that an item is not new (i.e., is either a current or a prior TBRI).

strength of current and lag 1 probes, with new probes at a distance from this cutoff. This suggests that response latency should be relatively short for new items (since they would be at a distance to produce fast no responses) and that decision time for current and prior items should be relatively long. The unprimed data displayed in Table 3 follow this pattern. For prior-only instructions, there would be two yes cutoffs, one between current and lag 1 probes, and another between lag 2 and new probes, and therefore, the latency distribution should tend to be flat and elevated. The data show that although RTs to lag 1, lag 2 , and new items are nearly identical, the RTs to current items under prior-only instructions are definitely shorter. Finally, inclusion instructions would put the decision threshold between lag 2 and new probes, which would tend to make current probes relatively fast. Table 3 does indeed show that RTs to current items under inclusion instructions are the shortest, with new items and lag 2 items the two slowest conditions, as would be expected if the response threshold were placed between the lag 2 and the new distributions. We anticipate, however, that we will need to gather more data before we could attempt a quantitative fit of the RT data.

In standard recognition memory designs, novel distractors have shorter RTs than either targets or related distractors (see Clark \& Gronlund, 1996, for a review). Such an effect is generally interpreted as evidence for recognition's being based solely on familiarity. The pattern of RT performance in the present experiment, however, is much more consistent with a trace comparison process in which novel distractors are far from the cut- offs in the exclusion task but close to the cutoff between prior lag 2 items and novel items that is required in the prior-only and inclusion conditions. ${ }^{4}$

\section{Testing the Models}

The maximum likelihood parameter estimates for the conjoint recognition model and the source-monitoring model are shown in Tables 4 and 5, respectively. Both models were fit by finding the model parameters that maximized the likelihood that the obtained data was generated by the model under consideration. A standard expectancy maximization algorithm (e.g., Hu \& Batchelder, 1994) was programmed to estimate the model parameters. ${ }^{5}$ The standard loglikelihood statistic $G^{2}$-which has a $\chi^{2}$ distribution - is reported as the measure of goodness of fit. For the fits reported here, $\alpha=.05$ is used as the threshold for the rejecting a model. When the priming variable is included, the experimental design provides 24 degrees of freedom in the data, and since the version of the conjoint recognition model used here has 18 parameters, 6 degrees of freedom remain for testing the fit of the model. The full conjoint recognition model fits the data $\left[G^{2}(6)=10.5\right]$. This value is below the threshold for rejecting the model, $\left[\chi^{2}(6)=12.6\right]$. Including the priming variable, the source-monitoring model has 22 parameters, leaving 2 for testing the fit of the model. The full sourcemonitoring model does not fit the data quite as well $\left[G^{2}(2)=6.6\right]$, being slightly above the threshold for rejection $\left[\chi^{2}(2)=6.0\right]$.

The hierarchical structure of the multinomial models makes it straightforward to test the null hypothesis that 
Table 6

Parameter Estimates for the Best-Fitting Conjoint Recognition Model

\begin{tabular}{lcccccccc}
\hline & $V_{\mathrm{C}}$ & $V_{\mathrm{L} 1}=V_{\mathrm{L} 2}$ & $G_{\mathrm{C}}$ & $G_{\mathrm{L} 1}$ & $G_{\mathrm{L} 2}$ & $B E$ & $B P$ & $B I$ \\
\hline $\begin{array}{l}\text { Unprimed } \\
\begin{array}{l}\text { Both } \\
\text { Primed }\end{array}\end{array}$ & .69 & .48 & .34 & .27 & .21 & .05 & .28 & .28 \\
\hline
\end{tabular}

Note- $V_{\mathrm{C}}$, probability of verbatim recognition of the current TBRI; $V_{\mathrm{L} 1}$ and $V_{\mathrm{L} 2}$, probability of verbatim recognition of prior TBRIs at lag 1 and lag 2; $G_{\mathrm{C}}$, probability of gist recognition of the current TBRI; $G_{\mathrm{L} 1}$ and $G_{\mathrm{L} 2}$, probability of gist recognition of the lag1 and lag 2 TBRIs; $B E$, probability of guessing "yes" under exclusion instructions; $B P$, probability of guessing "yes" under prior-only instructions; $B I$, probability of guessing "yes" under inclusion instructions.

Table 7

Parameter Estimates for the Best-Fitting Source-Monitoring Model

\begin{tabular}{lcccccccc}
\hline & $D_{\mathrm{C}}$ & $D_{\mathrm{L} 1}=D_{\mathrm{L} 2}$ & $S_{\mathrm{C}}$ & $S_{\mathrm{L} 1}$ & $S_{\mathrm{L} 2}$ & $B E$ & $B P$ & $B$ \\
\hline Unprimed & & & & & & & 1.0 & .27 \\
$\begin{array}{l}\text { Both } \\
\text { Primed }\end{array}$ & .79 & .61 & .86 & .76 & .83 & .15 & & \\
\hline
\end{tabular}

Note- $D_{\mathrm{C}}$, probability of distinguishing the current TBRI from a new item; $D_{\mathrm{L} 1}$ and $D_{\mathrm{L} 2}$, probability of distinguishing lag 1 and lag 2 TBRIs from new items; $S_{\mathrm{C}}$, probability of correctly identifying the current TBRI as current; $S_{\mathrm{L} 1}$ and $S_{\mathrm{L} 2}$, probability correctly identifying lag 1 or lag 2 items as prior; $B E$, probability of guessing "yes" under exclusion instructions when an old/new discrimination has not been made; $B P$, probability of guessing "yes" under prior-only instructions when an old/new discrimination has not been made; $B$, probability of guessing that an item is not new (i.e., is either a current or prior TBRI). Parameters that do not differ significantly as a function of priming are entered in the row labeled "Both." Note that the parameters $A E$ and $A P$ do not appear in the best-fitting version of the model, since their values did not significantly differ from 1.0.

any chosen set of parameters are equivalent. Equating parameters always reduces the fit of the model (increases $G^{2}$ ), and if the increase in $G^{2}$ exceeds the value that represents the upper .05 of the $\chi^{2}$ distribution, the parameters are assumed to differ significantly. If $G^{2}$ does not exceed this threshold, the parameters are not assumed to differ significantly. In this way, an attempt was made to find the models with the fewest number of parameters that still fit the data.

Table 6 shows the minimum number of parameters necessary for the conjoint model to fit the accuracy data. The reduction in parameters from 18 to 8 leaves 16 degrees of freedom for testing the fit of the model. The reduced version of the conjoint recognition model provides an excellent fit to the data $\left[G^{2}(16)=14.1, \chi^{2}(16)=\right.$ 26.3]. Notably, only the guessing parameter has to be adjusted to fit the effects of the priming variable, and that only in the inclusion instruction condition, where participants were instructed to respond "yes" if the item had ever appeared in the experiment. An increase in the bias to say "yes" to primed items was observed in that condition $\left[G^{2}(1)=32.2 ; \chi^{2}(1)=3.8\right]$.

An examination of the parameter estimates with respect to the verbatim trace shows that the value of the $V_{\mathrm{C}}$ parameter, the probability of identifying the current TBRI, is significantly greater than that of $V_{\mathrm{L} 1}$ and $V_{\mathrm{L} 2}$, the probability of identifying a prior trace as distinct from the current trace $\left[G^{2}(1)=39.0 ; \chi^{2}(1)=3.8\right]$. There is no significant difference between the identity parameters $V_{\mathrm{L} 1}$ and $V_{\mathrm{L} 2}$ across the two lags. As was predicted, the gist trace differs significantly as a function of lag $\left[G_{\mathrm{C}}>G_{\mathrm{L} 1}>G_{\mathrm{L} 2}\right.$, $\left.G^{2}(2)=6.5, p<.05 ; \chi^{2}(2)=6.0\right]$. The decrease in verbatim recognition from $V_{\mathrm{C}}$ to $V_{\mathrm{L} 1}$ is .21 , which is much greater than the decrease in gist recognition from $G_{C}$ to $G_{\mathrm{L} 1}$, which is .07. As can be seen in Table 4 the full conjoint model confirms these trends and shows no evidence of a priming effect on the two parameter sets representing verbatim and gist processing.

Although the full source-monitoring model did not provide as good a fit to the data as the conjoint recognition model, we proceeded to find the best-fitting version of the model, to discover whether it would provide a similar interpretation of the experimental results. The reduced version of the source-monitoring model has $10 \mathrm{pa}-$ rameters, leaving 14 degrees of freedom for testing the fit of the model. As is shown in Table 7, similar conclusions would result from the best-fitting source-monitoring model, which also fits the data $\left[G^{2}(14)=12.8 ; \chi^{2}(14)=\right.$ 23.7 ; this fit is qualified by the earlier lack of fit, however]. As is the case for the conjoint recognition model, the only effects of priming are seen in the guessing parameters: BP (bias to identify an item as prior) is higher in the unprimed than in the primed condition $\left[G^{2}(1)=\right.$ $10.3 ; \chi^{2}(1)=3.8$ ], and; B (bias to call an item "old") is higher in the primed than in the unprimed condition $\left[G^{2}(1)=27.1 ; \chi^{2}(1)=3.8\right]$. Similar to the results for the conjoint recognition verbatim parameter $V$, the item recognition parameter $D$ is higher for current items than for prior items but does not differ between lag 1 and lag $2\left[G^{2}(1)=57.5 ; \chi^{2}(1)=3.8\right]$. The source identification 
parameter $S$ differs as a function of lag $\left[G^{2}(2)=6.2\right.$; $\left.\chi^{2}(2)=6.0\right]$, indicating that the current and lag 2 items are more easily classified as current or prior, respectively, than are lag 1 items.

\section{DISCUSSION}

In the experiment reported here, a modified version of Brainerd et al.'s (1999) conjoint recognition model was used to examine the results of a recognition version of the classic Brown-Peterson STM paradigm. The model, based on Reyna and Brainerd's (1995) fuzzy trace theory, assumes that recognition judgments either are made as the result of a verbatim comparison of the recognition item to the original item or are the result of the similarity of the gist of the recognition item and the original item. Importantly, the conjoint recognition model fits the results of this experiment, which manipulated similarity in environmental/temporal context and minimized semantic similarity, which has been the typical experimental and theoretical interpretation of gist. Specifically, the model parameter representing the probability that a correct response to a recognition probe was based on the gist memory trace decreases monotonically from the case in which the recognition probe is from the current trial to the case in which the probe is from the immediately previous trial (lag 1) or from the trial before the previous trial (lag 2). This is to be expected if environmental context fluctuates over time. The data clearly are consistent with a model that attributes interference in the recognition form of the Brown-Peterson paradigm to changes in the context that render a contextually marked memory less retrievable over time and less similar to recognition probes presented in the current context. Clearly, the verbatim memory trace becomes less accessible as context shifts over time as well, and the model parameter representing the probability of a correct response based on a verbatim comparison of the probe and the original item rapidly decreases as a function of lag. As is predicted by fuzzy trace theory, the probability of an accurate response based on the verbatim trace decreased much more between current probes and lag 1 probes than did the probability of an accurate response based on the gist trace.

The analysis based on the conjoint recognition model served to untangle a three-way interaction in the accuracy data. As we anticipated, the nature of the task requirements under the exclusion and prior-only instructions limited the effect of priming on response bias to the inclusion condition only. The only parameter of the model affected by the priming manipulation was the parameter representing response bias in the inclusion conditions. No other parameters of the model showed an effect of priming. Priming the probes just prior to their presentation did not affect performance when the participants were required to discriminate between recently presented and, therefore, roughly equally salient items, as was the case under the exclusion and prior-only instructions. The prime affected performance only when the participants were required under inclusion instructions to discriminate between previously presented items and novel distractors, but not between the previously presented items themselves. No evidence was obtained for any additional attachment of context to the trace probed under the priming condition, since there was no significant main effect of priming on response accuracy and the verbatim and gist memory parameters did not significantly differ between the primed and the unprimed experimental conditions.

An additional analysis of the results, using Batchelder and Reifer's (1990) source-monitoring model provides further support that priming the recognition probe affects participants' response bias but has little or no effect on memory traces. It is notable that both of the two different multinomial models employed in our investigation were consistent with the same explanation. The sourcemonitoring model is intended to analyze the ability of participants to correctly identify the source of their knowledge about a particular memory probe. Our participants were not explicitly instructed to identify the source of the recognition probe, so perhaps participants can implicitly instruct themselves to examine the source of their familiarity with a memory probe.

Moreover, we were able to modify the conjoint recognition procedure to fit the Brown-Peterson task and demonstrate that the gist parameter can be related to the task demands. Our modified instructions focused on the contextual similarity (had the item ever occurred in the experiment and when [current trial, previous trial, or either]) instead of semantic similarity. The gist parameter may be seen as representing a general similarity dimension.

Brainerd et al.'s (1999) conjoint recognition approach seems to us to offer a very useful tool for further research. Specifically, much of the earlier work with the model has focused on the role of semantic similarity and its influence on the gist parameter. A number of studies of the Deese/ Roediger-McDermott false memory paradigm have successfully utilized the instructions presented by Brainerd et al. (e.g., Brainerd, Payne, Wright, \& Reyna., 2003). It is notable that we were able to present different instructions prior to the task without notably altering the pattern of results. Further research employing categorized words, in the manner of Loess (1967), with the revised procedure will enable us to evaluate the source of trace confusability in proactive interference paradigms by essentially combining semantic and contextual manipulations of gist. We believe that the extension of the methodology proposed by Brainerd et al. can prove fruitful, enabling us to return to old unsolved problems, such as the locus of interference in the Brown-Peterson task, and perhaps, in the long run, to the important problem of the determinants of decision latency in a variety of recognition memory tasks.

\section{REFERENCES}

Batchelder, W. H., \& Riefer, D. M. (1990). Multinomial processing models of source monitoring. Psychological Review, 97, 548-564. BAtChelder, W. H., \& Riefer, D. M. (1999). Theoretical and empiri- 
cal review of multinomial process tree modeling. Psychonomic Bulletin \& Review, 6, 57-86.

BENNETT, R. W. (1975). Proactive interference in short-term memory: Fundamental forgetting processes. Journal of Verbal Learning \& Verbal Behavior, 14, 123-144.

Bernstein, I. H., \& Welch, K. (1991). Awareness, false recognition, and the Jacoby/Whitehouse effect. Journal of Experimental Psychology: Human Perception \& Performance, 120, 324-328.

Bowers, J. S. (1999). Priming is not all bias: Commentary on Ratcliff and McKoon (1997). Psychological Review, 106, 582-596.

Brainerd, C. J., Payne, D. G., Wright, R., \& Reyna, V. F. (2003). Phantom recall. Journal of Memory \& Language, 48, 445-467.

Brainerd, C. J., Reyna, V. F., \& KneER, R. (1995). False-recognition reversal: When similarity is distinctive. Journal of Memory \& Language, 34, 157-185.

Brainerd, C. J., Reyna, V. F., \& Mojardin, A. H. (1999). Conjoint recognition. Psychological Review, 106, 160-179.

Brown, J. A. (1958). Some tests of the decay theory of immediate memory. Quarterly Journal of Experimental Psychology, 10, 12-21.

Brown, V. (1998). Comparing parallel and sequential multinomial models of letter identification. In C. Dowling, F. Roberts, \& P. Theuns (Eds.), Recent progress in mathematical psychology (pp. 253284). Mahwah, NJ: Erlbaum.

Clark, S. E., \& Gronlund, S. D. (1996). Global matching models of recognition memory: How the models match the data. Psychonomic Bulletin \& Review, 3, 37-60.

CROWDER, R. G., \& GREENE, R. L. (1987). The context of remembering: Comments on the chapters by Glenberg, Gorfein, and Wickens. In D. S. Gorfein \& R. R. Hoffman (Eds.), Memory and learning: The Ebbinghaus centennial conference (pp. 191-199). Hillsdale, NJ: Erlbaum.

Estes, W. K. (1950). Toward a statistical theory of learning. Psychological Review, 57, 94-107.

FiSHER, D. F., JAROMBEK, J. J., \& KARSH, R. (1974). Short-term memory (1958-1973): An annotated bibliography. Aberdeen Proving Ground, MD: Human Engineering Laboratory.

GlenberG, A. M. (1987). Temporal context and recency. In D. S. Gorfein \& R. R. Hoffman (Eds.), Memory and learning: The Ebbinghaus centennial conference (pp. 173-190). Hillsdale, NJ: Erlbaum.

GorfEIN, D. S. (1987). Explaining context effects on short-term memory. In D. S. Gorfein \& R. R. Hoffman (Eds.), Memory and learning: The Ebbinghaus centennial conference (pp. 153-172). Hillsdale, NJ: Erlbaum.

Gorfein, D. S., \& JaCobson, D. E. (1972). Proactive effects in shortterm recognition memory. Journal of Experimental Psychology, 95, 211-214.

Gorfein, D. S., \& JACOBSon, D. E. (1973). Memory search in a BrownPeterson short-term memory paradigm. Journal of Experimental Psychology, 99, 82-87.

Hu, X., \& BATChELDER, W. H. (1994). The statistical analysis of general processing tree models with the EM algorithm. Psychometrika, $\mathbf{5 9}, 21-47$.

JACOBY, L. L. (1991). A process dissociation framework: Separating automatic from intentional uses of memory. Journal of Memory \& Language, 30, 513-541.

JaCOBY, L. L., \& Whitehouse, K. (1989). An illusion of memory: False recognition influenced by unconscious perception. Journal of Experimental Psychology: General, 118, 126-135.

JoHnson, M. K., \& RAYE, C. L. (1981). Reality monitoring. Psychological Review, 88, 67-85.

LoEss, H. (1967). Short-term memory: Word class and sequence of items. Journal of Experimental Psychology, 118, 126-135.

MANDleR, G. (1980). Recognizing: the judgment of previous occurrence. Psychological Review, 87, 252-271.

Peterson, L. R., \& Peterson, M. J. (1959). Short-term retention of individual items. Journal of Experimental Psychology, 58, 193-198.

RATCLIFF, R., \& MCKoon, G. (1997). A counter mode for implicit priming in perceptual word identification. Psychological Review, 104, 319-343.
Reyna, V. F., \& Brainerd, C. J. (1995). Fuzzy-trace theory: An interim synthesis. Learning \& Individual Differences, 7, 1-75.

Riefer, D. M., \& BATCHELDER, W. H. (1988). Multinomial modeling and the measurement of cognitive processes. Psychological Review, 95, 318-339.

SCHNEIDER, W. (1988). Micro Experimental Laboratory: An integrated system for IBM-PC compatibles. Behavior Research Methods, Instruments, \& Computers, 20, 206-217.

Wickens, D. D., Born, D. G., \& Allen, C. K. (1963). Proactive inhibition and item similarity in short-term memory. Journal of Verbal Learning \& Verbal Behavior, 2, 440-445.

Zeelenberg, R., Wagenmakers, E. M., \& Raaijmakers, J. G. W. (2002). Priming in implicit memory tasks: Prior study causes enhanced discriminality, not only bias. Journal of Experimental Psychology: General, 131, 38-47.

\section{NOTES}

1. The probability predicted by the model of a particular response in a given experimental condition is determined by multiplying the parameters along the branches of the tree that lead to the response and then summing over each occurrence of the response in the selected condition. For example, the predicted probability of responding "yes" when the recognition probe is a current TBRI under exclusion instructions is $V_{\mathrm{C}}+\left(1-V_{\mathrm{C}}\right) * G_{\mathrm{C}}+\left(1-V_{\mathrm{C}}\right) *\left(1-G_{\mathrm{C}}\right) * B E$. That is, a "yes" response in the exclusion condition can occur in three mutually exclusive ways: retrieval of the verbatim trace of the current TBRI with a probability of $V_{\mathrm{C}}$ or, failing that, retrieval of the gist trace with a probability of $\left(1-V_{\mathrm{C}}\right) * G_{\mathrm{C}}$, or lastly, failing that, the participant may guess "yes" with a probability of $\left(1-V_{\mathrm{C}}\right) *\left(1-G_{\mathrm{C}}\right) * B E$. Note that since there are only two possible responses in each condition, it is always the case that $P($ "no") $=1-P$ ("yes").

2. It should be noted that the verbatim recognition parameters $V_{\mathrm{C}}$, $V_{\mathrm{L} 1}$, and $V_{\mathrm{L} 2}$ have a slightly different interpretation than the corresponding parameters $I_{\mathrm{t}}$ and $N_{\mathrm{r}}$ in Brainerd et al.'s (1999) original conjoint recognition model. In the original model, $I_{\mathrm{t}}$ represents the probability that a recognition probe identical to the original item, will cue the recall of the verbatim trace of the original item leading to a correct response (called an identity judgment). In the Brown-Peterson recognition task modeled here, the verbatim recognition parameter $V_{\mathrm{C}}$ has a similar meaning; it represents the probability that when the recognition probe is the current TBRI, retrieval of the current TBRI's verbatim trace leads to a correct response. On the other hand, the parameter $N_{\mathrm{r}}$ in the original conjoint recognition model represents the probability that a recognition probe semantically related to the original item leads to the retrieval of the original verbatim trace and, therefore, to the recognition that the probe and the original item are, in fact, different (called a nonidentity judgment). In Brainerd et al.'s (1999) conjoint recognition tasks, the related recognition probes do not appear on the original list of study items. However, in the Brown-Peterson recognition task, the parameters $V_{\mathrm{L} 1}$ and $V_{\mathrm{L} 2}$ - which are the counterparts to $N_{\mathrm{r}}$ in the original modelrepresent the probability that recognition probes identical to lag 1 or lag 2 TBRIs lead to retrieval of the verbatim traces of the original lag 1 or lag 2 TBRIs. That is, the fact that prior probes are items that have actually appeared in the experiment leads to identity judgments for both current and prior probes.

3. We thank Charles Brainerd for pointing this out in his review of an earlier version of this article.

4. We again thank Charles Brainerd for pointing this out in his review of an earlier version of this article.

5 . The algorithm typically converged to a solution from a set of random initial values in less than 70 iterations for the conjoint recognition model and less than 180 iterations for the source-monitoring model.

(Manuscript received July 23, 2003 revision accepted for publication November 14, 2003.) 